\title{
Genetic Basis of Ventricular Arrhythmias
}

\author{
Raha Pazoki • Arthur A. M. Wilde • Connie R. Bezzina
}

Published online: 3 September 2010

(C) The Author(s) 2010. This article is published with open access at Springerlink.com

\begin{abstract}
Sudden cardiac death (SCD) is a leading cause of total and cardiovascular mortality, and ventricular fibrillation is the underlying arrhythmia in the majority of cases. In the young, where the incidence of SCD is low, a great proportion of SCDs occur in the context of inherited disorders such as cardiomyopathy or primary electrical disease, where a monogenic hereditary component is a strong determinant of risk. Marked advancement has been made over the past 15 years in the understanding of the genetic basis of the primary electrical disorders, and this has had an enormous impact on the management of these patients. At older ages, the great majority of SCDs occur in the context of acute myocardial ischemia and infarction. Although epidemiologic studies have shown that heritable factors also determine risk in these cases, inheritance is likely complex and multifactorial, and progress in understanding the genetic and molecular mechanisms that determine susceptibility to these arrhythmias, affecting a greater proportion of the population, has been very limited. We review the most recent insights gained into the genetic basis of both the monogenic and the more complex ventricular arrhythmias.
\end{abstract}

\author{
R. Pazoki • A. A. M. Wilde · C. R. Bezzina \\ Department of Clinical and Experimental Cardiology, \\ Heart Failure Research Center, \\ Amsterdam, The Netherlands \\ R. Pazoki \\ Department of Clinical Epidemiology Biostatistics \\ and Bioinformatics, Academic Medical Center, \\ Amsterdam, The Netherlands \\ C. R. Bezzina $(\square)$ \\ Department of Experimental Cardiology, \\ Academic Medical Center, \\ Room L2-108-1 Meibergdreef 9, \\ 1105 AZ Amsterdam, The Netherlands \\ e-mail: C.R.Bezzina@amc.uva.nl

Keywords Genetics · Genetic polymorphisms · Genome-wide association study · Mutation · Cardiac arrhythmias $\cdot$ Ventricular fibrillation $\cdot$ Sudden cardiac death

\section{Introduction}

Sudden cardiac death (SCD) accounts for $15 \%$ to $20 \%$ of all natural deaths in adults in the United States and Western Europe, and up to $50 \%$ of all cardiovascular deaths [1]. Ventricular fibrillation is the most common underlying cause [2]. Ventricular arrhythmias present with various symptoms including palpitations, chest pain, or syncope and may occur in various pathologic settings such as cardiomyopathies, congenital heart disease, inflammatory myocardial disease, as well as in the structurally normal heart. SCD is rare among young individuals. In individuals younger than 40 years of age, incidence of sudden death is approximately 1.3 to 8.5 per 100,000 person-years, and the vast majority of cases are considered to be $\operatorname{SCD}[3,4]$. In this age group, a great proportion of SCDs occur in the context of potentially inherited disease such as cardiomyopathy and primary electrical disease $[5,6]$, and a strong monogenic hereditary component is thought to determine risk of sudden death in most cases [7, 8]. In contrast, at older ages, the great majority of SCDs occur in the context of acute myocardial ischemia/ infarction [9]. Epidemiologic studies have shown that heritable factors also determine risk in these cases [10-12].

We review recent insights gained into the genetics of ventricular arrhythmias. We provide a brief update on novel genes uncovered for the monogenic primary electrical disorders and highlight results of recent genome-wide association studies that have uncovered novel genetic loci that may contribute to risk of SCD in the general population. 


\section{Monogenic Primary Arrhythmia Syndromes}

Molecular genetic research into the monogenic arrhythmia syndromes over the past 15 years has had an enormous impact on the management of patients with these disorders [13]. They are now known to be caused primarily by mutations in genes encoding ion channel subunits or their regulatory components. The increased understanding of the genetic basis of these disorders has provided great insight into many aspects of these disease entities, including their pathophysiology, and prognosis, and optimal treatment options for the specific molecular genetic subtypes. Perhaps the most significant impact has been on the management of patients with the congenital long QT syndrome (LQTS). This disorder is among the most prevalent of the monogenic arrhythmia syndromes and has facilitated large studies looking into genotype-phenotype relationships. Such studies have uncovered triggers specific to the different genetic subtypes, providing opportunities for lifestyle adjustments and an explanation as to why $\beta$-blockers might not be equally effective in all genetic subtypes (i.e., they are most effective in LQTS1 subtype and of high to moderate effectiveness in the LQTS2 subtype, in accordance with the adrenergic triggers in these subtypes, and of uncertain effectiveness in LQTS3 [14, 15]. Knowledge of the genetic subtype may therefore be critical to the cardiologist in assessing optimal treatment strategies.

The availability of a genetic test has also provided an opportunity for presymptomatic diagnosis of patients with these disorders. Active cascade screening within families is generally advised after the identification of an affected proband because it leads to timely presymptomatic treatment of relatives also carrying the mutation, which may be life-saving, whereas relatives not carrying the genetic defect can be reassured [16॰]. However, genetic testing is not always straightforward, and a proper interpretation of the genetic test result is critical considering the implications of mutation identification. The occurrence of a mutation within a likely gene does not automatically imply genetic causation. Analysis for co-segregation with the disorder in an extended pedigree is often helpful in establishing causality of an identified mutation, but such pedigrees are not always available. Distinguishing pathogenic mutations from innocuous and clinically silent gene variants remains a major challenge in many instances. Mutation type (e.g., nonsense vs. missense), mutation location in channel subdomain (e.g., pore vs. transmembrane vs. linker), and ethnic-specific background rates have been shown to be critical factors in predicting the pathogenicity of novel mutations [17•]. For instance, in LQTS, a novel mutation (an unclassified variant) identified in the transmembrane regions of the $S C N 5 A$-encoded sodium channel is much more likely to be pathogenic than when located in an interdomain linker [17•]. Thus, genetic tests must be viewed as probabilistic tests to be interpreted along with other diagnostic tests.

In the research setting, the identification of novel genes underlying the monogenic rhythm disorders may be rather challenging. Ideally for such studies, one has access to genetic material from multiple clinically affected individuals within an extended pedigree with the possibility of establishing genetic linkage to a particular chromosomal segment followed by the sequencing of candidate genes for identification of the causal defect. However, availability of an extended pedigree is rarely the case for these disorders in particular because individuals within a pedigree might have already died suddenly of arrhythmia before DNA collection is possible. Furthermore, as is the case for most Mendelian monogenic disorders, these disorders display reduced penetrance (not all mutation carriers have clinical signs of the disorder) and variable clinical expression [18, 19], which further complicates the process of gene identification. One group of rhythm disorders for which it is exceptionally difficult to track the genetic substrate is idiopathic ventricular fibrillation (VF), defined as spontaneous VF in the absence of identifiable structural or electrical heart disease [20]. The diagnosis of this disorder, which accounts for as many as $10 \%$ of sudden deaths, mainly in the young, cannot be made on the basis of electrocardiogram (ECG) abnormalities but can only be made after the occurrence of (aborted) SCD. Many affected patients die young, thus leaving only small numbers of patients and material available for analysis. Using an alternative approach, searching for shared ancestral haplotypes (chromosomal segments) among three distantly related pedigrees with the disorder originating from one region in the Netherlands, our group recently implicated the DPP6 gene in this disorder [21••]. DPP6 encodes dipeptidyl-peptidase 6, a putative subunit of the transient outward current potassium-channel complex $\left(I_{\mathrm{To}}\right)$ [22]. Although, expression of DPP6 was increased in cardiac biopsies of carriers of the risk haplotype [21••], more research is required to establish the pathophysiologic mechanism of DPP6-related idiopathic VF and how such a genetic defect is silent on ECG. Genetic testing is crucial in these patients because it is the only means of identifying those at risk of developing potentially fatal arrhythmia allowing for timely presymptomatic implantation of an implantable cardioverter defibrillator.

A disorder that has attracted much attention in recent months is the Early Repolarization Syndrome. For a long time, early repolarization, consisting of an elevation of the QRS-ST junction (J point), QRS notching or slurring (J wave), and a tall symmetric $\mathrm{T}$ wave, was considered to be a benign feature [23]. Three case-controls studies however recently demonstrated that a pattern of early repolarization in the inferior and/or lateral leads was more frequent in patients with idiopathic VF compared with controls [24••, 25••, 26]. A 
community-based study in Finland also showed that an early repolarization pattern in the inferior leads of the ECG is associated with an increased risk of death from cardiac causes during very long-term follow-up in middle-aged individuals [27]. So far, only one mutation in the KCNJ8 gene, encoding a subunit of the $\mathrm{K}_{\mathrm{ATP}}$ channel, has been reported for the disorder in a single patient [28]. It is expected that unraveling the genetic basis of this newly recognized disease entity will aid in the understanding of the mechanism for this ECG pattern and related arrhythmia.

\section{Arrhythmias with a More Complex Inheritance Pattern}

In recent years, interest in the genetics of cardiac arrhythmias has shifted to include the search for those genetic factors influencing risk of SCD in the general adult population. In adults, the overwhelming majority $(\sim 80 \%)$ of SCDs is caused by the sequela of coronary artery disease, namely myocardial ischemia or acute myocardial infarction (MI) $[9,29]$, where SCD is the first clinically identified expression of heart disease in up to one half of cases [30].

In the late 1990s, two important studies advanced the concept that even in these more common arrhythmias, a genetic component also contributes to risk. In a populationbased case-control study, a family history of MI or SCD, after correction for all common risk factors, was positively associated with the risk of SCD [10]. In the Paris Prospective Study, selectively performed in men, among whom 118 cases of sudden death occurred, parental sudden death was found to be an independent risk factor for sudden death [11]. A study from our group investigated this concept further in the Arrhythmia Genetics in the Netherlands Study (AGNES), conducted specifically in patients with a first acute MI [12]. In this study we demonstrated that familial sudden death occurred significantly more frequently among patients with a first MI complicated by VF (cases) compared with patients presenting with a first MI but without VF (controls) [12]. However, in contrast to the significant advances made in the understanding of the genetics of the monogenic arrhythmia syndromes, progress in understanding the genetic and molecular mechanisms that determine susceptibility to these common arrhythmias, affecting a much greater proportion of the population, has been limited [31-33]. An important reason for this slow progress is the fact that most victims die outside of the hospital, making it extremely difficult to include patients with appropriately consented DNA samples for genetic studies.

In an effort to identify common genetic variation within the genome contributing to risk of VF during acute MI, our group recently conducted a genomewide association study for VF in the AGNES population [34•.0]. By comparing the frequency of common single nucleotide polymorphisms
(SNPs) spread throughout the 22 autosomes between MI patients with VF (cases) and MI patients without VF (controls) from the AGNES study, we identified a region on chromosome 21 in the vicinity of the CXADR gene associated with susceptibility to VF. $C X A D R$ encodes the coxsackievirus and adenovirus receptor (CAR) protein, which has a long-recognized role as viral receptor in the pathogenesis of viral myocarditis and its sequela of dilated cardiomyopathy $[35,36]$. Interestingly, the frequency of active coxsackie $\mathrm{B}$ virus infection has been reported to be high in a group of MI patients who died suddenly [37]. Two studies have reported a physiologic role for the receptor in localization of connexin 45 at the intercalated disks of the cardiomyocytes in the atrioventricular node, and a role in conduction of the cardiac impulse within this cardiac compartment $[38 \bullet, 39 \bullet$. Thus, CXADR can be considered a very relevant candidate gene for the association detected at this locus.

As a complementary approach to establishing direct bridges between genetic variation and arrhythmia susceptibility, researchers in the field have also undertaken a strategy whereby SNPs are first analyzed for effects on heart rate and other ECG indices of conduction and repolarization. This approach stems from knowledge that these ECG measures constitute heritable traits $[40,41]$ and that their extremes (too long or too short) influence risk of arrhythmia, both in the general population $[42,43]$ as well in specific disease groups [44], which makes ECG indices potentially relevant intermediate phenotypes. The most extensively studied ECG parameter studied until now in this way has been the QT interval, reflecting ventricular repolarization. These studies have uncovered numerous genetic loci and SNPs modulating this measure (Table 1), the most significant of which have consistently been SNPs in and around the NOSIAP gene, encoding the nitric oxide synthase 1 (neuronal) adaptor protein $[45,46 \bullet, 47 \bullet]$. Although the exact mechanism for the effect of SNPs in NOS1AP on ventricular repolarization is still unknown, one must realize that this gene was previously unlinked to cardiac electrophysiology, underscoring the power of the genome-wide association approach to highlight unknown pathways that could represent an important means to ultimately unravel mechanisms of disease and development of new therapies.

Crotti et al. [48•] and Tomás et al. [49•] went on to test whether SNPs in NOS1AP could modulate disease expression (QTc and arrhythmia) in the congenital LQTS, where pronounced inter-individual variability in QTc-prolongation and occurrence of arrhythmia exists [19]. The first study investigated South African families segregating a founder mutation in $K C N Q 1$ (all affected individuals were carriers of the $\mathrm{A} 341 \mathrm{~V}$ mutation in $K C N Q 1$ ) and demonstrated that carriers of risk alleles at NOS1AP SNP sites had longer QTc and an increased risk of cardiac arrest and SCD. Carrying out 
Table 1 Candidate genes identified in genome-wide association studies for heart rate and ECG indices of conduction (PR, QRS) and repolarization (QTc) displaying association at genome-wide significance $\left(P<5 \times 10^{-8}\right)$ are listed

$E C G$ electrocardiogram

\begin{tabular}{|c|c|c|c|}
\hline QT interval & PR interval & QRS interval & Heart rate \\
\hline$A T P 1 B 1[47 \bullet]$ & ARHGAP24 $[51 \bullet, 53 \bullet]$ & $C A V 1[51 \bullet]$ & MYH6 [51•] \\
\hline GINS3 [46•] & $C A V 1 / C A V 2[51 \bullet, 53 \bullet]$ & $C D K N 1 N[51 \bullet]$ & \\
\hline KCNE1 [46•] & MEIS1 [53•] & $D K K 1[51 \bullet]$ & \\
\hline $\mathrm{KCNH} 2[46 \bullet, 47 \bullet]$ & MYH6 [51•] & $S C N 10 A[52 \bullet]$ & \\
\hline KCNJ2 [47•] & $N K X 2-5[53 \bullet]$ & $T B X 5[51 \bullet]$ & \\
\hline$K C N Q 1[46 \bullet, 47 \bullet]$ & $\operatorname{SCN10A}[51 \bullet, 52 \bullet, 53 \bullet]$ & & \\
\hline$L I G 3-R F F L[46 \bullet]$ & $S C N 5 A[53 \bullet]$ & & \\
\hline $\operatorname{LITAF}[46 \bullet, 47 \bullet]$ & $\operatorname{SOX5}[53 \bullet]$ & & \\
\hline$N D R G 4[46 \bullet, 47 \bullet]$ & $T B X 5-T B X 3[51 \bullet, 53 \bullet]$ & & \\
\hline $\operatorname{NOS} 1 A P[45,46 \bullet, 47 \bullet]$ & $W N T 1 L[53 \bullet]$ & & \\
\hline \multicolumn{4}{|l|}{$P L N[46 \bullet, 47 \bullet]$} \\
\hline \multicolumn{4}{|l|}{ RNF207 [46•, 47•] } \\
\hline $\operatorname{SCN} 5 A[46 \bullet, 47 \bullet]$ & & & \\
\hline
\end{tabular}

such studies in the setting of families with a founder mutation is attractive because it circumvents the interindividual variability in disease manifestations that could otherwise arise as a consequence of the different primary genetic defects among study participants. In the second study, Tomás et al. [49•], who carried out association studies of NOS1AP SNPs in a large LQT patient cohort (901 patients from 520 families) with mutations in different genes, subsequently demonstrated that the effects of SNPs in NOS1AP are also detectable in a more diverse LQT patient cohort. These authors proposed that genotyping NOS1AP SNPs could in the future become useful in refining risk stratification in this disorder. However, the situation remains rather complex. One NOS1AP SNP (rs10494366) associated with risk of cardiac events in the LQTS study by Tomás et al. [49•] was not associated with risk of SCD in communitybased populations [50]. Furthermore, in the latter study, another SNP (rs12567209), which was not correlated with QT interval, was still associated with SCD, suggesting that there may be multiple functional elements within the NOS1AP region, some of which may modulate risk of arrhythmia independently from the repolarization process. Research is still required to resolve the underlying issue.

Following successful identification of several loci impacting the QT interval, investigators also turned their attention to other ECG parameters, namely heart rate [51•], the PR-interval (a measure of the time required for the electrical impulse to travel from the sinus node, through the atria and atrioventricular node to the Purkinje fibers) [51•, 52•, 53•], and QRS duration (a measure of the time required for depolarization of the ventricles) [51•]. These studies presented compelling evidence that SNPs in the SCN5A and $S C N 10 A$ genes modulate cardiac conduction. This finding is not surprising with respect to SNPs in $S C N 5 A$, which encodes the major sodium channel in the heart (Nav1.5). Sodium ion influx through this channel mediates the rapid upstroke of the cardiac action potential and is therefore a critical mediator of cardiac conduction, and mutations in SCN5A cause cardiac conduction disease [54]. On the other hand, the implication of the SCN10A gene (located within $<100 \mathrm{~kb}$-pairs of SCN5A on chromosome 3) as a modulator of cardiac conduction was a very novel finding. Note that SCN10A variant presented to be independent of SCN5A variant. SCN10A encodes the sodium channel Nav1.8, expressed primarily in the peripheral sensory nervous system and to a lesser extent in the heart. It has been hypothesized that amino acid-altering SNPs in SCN10A might be responsible for the observed effect on cardiac conduction [52•, 53•]. The exact mechanism however is still unknown. The A-allele at SNP rs6795970, which is associated with slower conduction in the general population, appeared protective against risk of VF in the setting of acute MI in the AGNES study [52•].

These genome-wide association studies have also uncovered SNPs in or near genes encoding transcription factors involved in cardiac development, including $N K X 2-5$ which encodes the cardiac-specific homeobox transcription factor Nkx2.5. Mutations in this gene were previously linked to atrial septal defect with conduction defects, tetralogy of Fallot, and high-degree atrioventricular block [55]. Another locus identified as impacting on conduction is in the region of the $T B X 3$ and $T B X 5$ genes, which encode T-box-containing transcription factors important for cardiac conduction system formation in the developing heart [56, 57]. Mutations in $T B X 5$ cause Holt-Oram syndrome, which includes atrial and ventricular septal defects, conduction disease, and occasionally atrial fibrillation [58], whereas mutations in $T B X 3$ cause ulnar-mammary syndrome, with limb, mammary, tooth, genital, and cardiac abnormalities [59]. Furthermore, as for genomewide association studies for QT interval, genomewide association studies for heart rate and conduction indices have also uncovered genes 
previously unlinked to cardiac electrical function (e.g., the association of ARHGAP24, which encodes a Rho-GTPaseactivating protein, with PR, and the association of MYH6, encoding the alpha heavy chain subunit of cardiac myosin., with heart rate and PR), thereby potentially illuminating novel pathways.

The wealth of information being generated by genomewide association studies is likely to trigger researchers around the world into following new avenues for research into novel pathways that could be involved in cardiac electrical function. However, one should realize that much work still needs to be done before we fully understand the exact molecular mechanisms whereby the identified genetic loci contribute to inter-individual variability in the related trait and ultimately to whether they also impact on risk of arrhythmia. Perhaps the most crucial of these issues relates to the fact that although these studies have uncovered regions on chromosomes that are linked to the traits of interest, some of which harbor highly plausible candidate genes, in basically all instances mentioned above, unequivocal evidence for genes mediating the observed effects is lacking, let alone knowledge of which functional genetic variants underlie these effects.

Another fact that needs mentioning is that the variants identified as modulators of ECG parameters, as expected in complex genetic traits, are associated with very small effect sizes. For instance, effect sizes for alleles impacting on QTc range from 1 to $3 \mathrm{~ms}$ per allele [46•,47•]. Even in aggregate, the identified genetic variants still explain only a very small percentage of the variance in these traits. In a meta-analysis by Pfeufer et al. [47•] including 16,678 individuals, association signals from 10 loci found to be associated with QTc-interval (at genome-wide statistical significance) in aggregate explained only $3.3 \%$ of the variance in QTc. In another meta-analysis performed by Newton-Cheh et al. [46•], comprising 13,685 individuals, in aggregate, $5.4 \%$ to $6.5 \%$ of the variation in QT interval was explained by 14 independent variants at 10 loci. Larger and larger association studies, including more study subjects, will be required to provide unequivocal evidence for novel genetic associations and complementary strategies to uncover the genetic underpinnings of these complex traits are obviously necessary. For instance, it is generally hypothesized that other genetic variants, such as rare variants not detected in genome-wide association studies that likely have stronger influences on these ECG indices, may be present.

\section{Conclusions}

The identification of the genetic defects underlying the monogenic arrhythmia syndromes is important because it provides insight into important aspects of these disease entities, including prognosis and optimal treatment options for the specific molecular genetic subtypes and allows for presymptomatic identification and treatment of patients at risk.

Recent genome-wide association studies have generated remarkable insight into chromosomal regions and genes that impact on cardiac electrical activity. Such strategies have now started to be applied to the identification of genes impacting on arrhythmia risk in the general population. These studies are likely to provide insight into pathways determining risk of arrhythmia in the general population.

Acknowledgment The authors are supported by research grants from the Netherlands Heart Foundation (2007B202), the InterUniversity Cardiology Institute of the Netherlands (ICIN, 06102), the Center for Translational Molecular Medicine of the Netherlands (CTMM-COHFAR) and the Leducq Foundation (05CVD). Connie R. Bezzina is an Established Investigator of the Netherlands Heart Foundation (2005T024).

Disclosure No potential conflicts of interest relevant to this article were reported.

Open Access This article is distributed under the terms of the Creative Commons Attribution Noncommercial License which permits any noncommercial use, distribution, and reproduction in any medium, provided the original author(s) and source are credited.

\section{References}

Papers of particular interest, published recently, have been highlighted as:

- Of importance

•• Of major importance

1. Myerburg RJ, Castellanos A: Cardiac arrest and sudden cardiac death. In Braunwald's Heart Disease: A Textbook of Cardiovascular Medicine, 8th edn. Edited by Libby P, Bonow RO, Mann DL, Zipes DP. Oxford: Elsevier; 2007.

2. Huikuri HV, Castellanos A, Myerburg RJ: Sudden death due to cardiac arrhythmias. N Engl J Med 2001, 345:1473-1482.

3. Liberthson RR: Sudden death from cardiac causes in children and young adults. N Engl J Med 1996, 334:1039-1044.

4. van der Werf C, Hofman N, Tan HL, et al.: The diagnostic yield in sudden unexplained death and aborted cardiac arrest in the young: the experience of a tertiary referral center in the Netherlands. Heart Rhythm 2009; doi:10.1016/j.hrthm.2010.05.03.

5. Corrado D, Basso C, Thiene G: Sudden cardiac death in young people with apparently normal heart. Cardiovasc Res 2001, 50:399-408.

6. Drory Y, Turetz Y, Hiss Y, et al.: Sudden unexpected death in persons less than 40 years of age. Am J Cardiol 1991, 68:1388-1392.

7. Tan HL, Hofman N, van Langen IM, et al.: Sudden unexplained death: heritability and diagnostic yield of cardiological and genetic examination in surviving relatives. Circulation 2005, 112:207-213.

8. Basso C, Carturan E, Pilichou K, et al.: Sudden cardiac death with normal heart molecular autopsy. Cardiovasc Pathol 2010 (in press). 
9. Zipes DP, Wellens HJ: Sudden cardiac death. Circulation 1998, 98:2334-2351.

10. Friedlander Y, Siscovick DS, Weinmann S, et al.: Family history as a risk factor for primary cardiac arrest. Circulation 1998, 97:155-160.

11. Jouven X, Desnos M, Guerot C, Ducimetiere P: Predicting sudden death in the population: the Paris Prospective Study I. Circulation 1999, 99:1978-1983.

12. Dekker LR, Bezzina CR, Henriques JP, et al.: Familial sudden death is an important risk factor for primary ventricular fibrillation: a casecontrol study in acute myocardial infarction patients. Circulation 2006, 114:1140-1145.

13. Wilde AA, Bezzina CR: Genetics of cardiac arrhythmias. Heart 2005, 91:1352-1358.

14. Schwartz PJ, Priori SG, Spazzolini C, et al.: Genotype-phenotype correlation in the long-QT syndrome: gene-specific triggers for life-threatening arrhythmias. Circulation 2001, 103:89-95.

15. Priori SG, Napolitano C, Schwartz PJ, et al.: Association of long QT syndrome loci and cardiac events among patients treated with beta-blockers. JAMA 2004, 292:1341-1344.

16. - Hofman N, Tan HL, Alders M, et al.: Active cascade screening in primary inherited arrhythmia syndromes: does it lead to prophylactic treatment? J Am Coll Cardiol 2010, 55:2570-2576. This retrospective study investigated the follow-up and treatment of mutation-positive relatives of probands with an inherited arrhythmia syndrome who were identified by active cascade screening in these families. This study showed that active cascade screening in families with LQTS, Brugada syndrome, and catecholamine-induced polymorphic ventricular tachycardia $(C P V T)$ resulted in immediate prophylactic treatment in a substantial proportion of mutation carriers, particularly in LQTS and $C P V T$.

17. - Kapa S, Tester DJ, Salisbury BA, et al.: Genetic testing for longQT syndrome: distinguishing pathogenic mutations from benign variants. Circulation 2009, 120:1752-1760. This study, which was carried out in 388 unrelated "definite" cases of LQTS and more than 1300 healthy controls, sought to quantify the value of mutation type and gene/protein region in determining the probability of pathogenicity for mutations in the most commonly affected LQTS genes (KCNQ1 [LQT1], KCNH2 [LQT2], and SCN5A [LQT3]).

18. Priori SG, Napolitano C, Schwartz PJ: Low penetrance in the long-QT syndrome: clinical impact. Circulation 1999, 99:529533.

19. Scicluna BP, Wilde AA, Bezzina CR: The primary arrhythmia syndromes: same mutation, different manifestations. Are we starting to understand why? J Cardiovasc Electrophysiol 2008, 19:445-452.

20. Survivors of out-of-hospital cardiac arrest with apparently normal heart. Need for definition and standardized clinical evaluation. Consensus Statement of the Joint Steering Committees of the Unexplained Cardiac Arrest Registry of Europe and of the Idiopathic Ventricular Fibrillation Registry of the United States. Circulation 1997, 95:265-272.

21. •- Alders M, Koopmann TT, Christiaans I, et al.: Haplotypesharing analysis implicates chromosome $7 \mathrm{q} 36$ harboring DPP6 in familial idiopathic ventricular fibrillation. Am J Hum Genet 2009, 84:468-476. In this study, haplotype-sharing analysis in three distantly related families identified an ancestral haplotype, including the DPP6 gene, that was shared among cases affected with idiopathic VF, implicating this gene in this disorder.

22. Radicke S, Cotella D, Graf EM, et al.: Expression and function of dipeptidyl-aminopeptidase-like protein 6 as a putative betasubunit of human cardiac transient outward current encoded by Kv4.3. J Physiol 2005, 565:751-756.
23. Wasserburger RH, Alt WJ.: The normal RS-T segment elevation variant. Am J Cardiol 1961, 8:184-192.

24. •- Haissaguerre M, Derval N, Sacher F, et al.: Sudden cardiac arrest associated with early repolarization. N Engl J Med 2008, 358:2016-2023. This study reviewed data from 206 cases who were resuscitated after cardiac arrest due to idiopathic VF and assessed the prevalence of electrocardiographic early repolarization. A group of 412 individuals without heart disease who were matched for age, sex, race, and level of physical activity were used as controls. This study showed that there is an increased prevalence of early repolarization among patients with a history of idiopathic $V F$

25. • Nam GB, Kim YH, Antzelevitch C: Augmentation of J waves and electrical storms in patients with early repolarization. $\mathrm{N}$ Engl $\mathrm{J}$ Med 2008, 358:2078-2079. This case-control study evaluated the incidence of early repolarization among 1395 controls who were representative of the general population and 15 patients classified as having idiopathic VF. An increased incidence of early repolarization was found in the latter group.

26. Rosso R, Kogan E, Belhassen B, et al.: J-point elevation in survivors of primary ventricular fibrillation and matched control subjects: incidence and clinical significance. J Am Coll Cardiol 2008, 52:1231-1238.

27. Tikkanen JT, Anttonen O, Junttila MJ, et al.: Long-term outcome associated with early repolarization on electrocardiography. $\mathrm{N}$ Engl J Med 2009, 361:2529-2537.

28. Haissaguerre M, Chatel S, Sacher F, et al. : Ventricular fibrillation with prominent early repolarization associated with a rare variant of KCNJ8/KATP channel. J Cardiovasc Electrophysiol 2009, 20:93-98.

29. Zipes DP, Camm AJ, Borggrefe M, et al.: ACC/AHA/ESC 2006 Guidelines for Management of Patients With Ventricular Arrhythmias and the Prevention of Sudden Cardiac Death: a report of the American College of Cardiology/American Heart Association Task Force and the European Society of Cardiology Committee for Practice Guidelines (writing committee to develop Guidelines for Management of Patients With Ventricular Arrhythmias and the Prevention of Sudden Cardiac Death): developed in collaboration with the European Heart Rhythm Association and the Heart Rhythm Society. Circulation 2006, 114:e385-e484.

30. Myerburg RJ: Sudden cardiac death: exploring the limits of our knowledge. J Cardiovasc Electrophysiol 2001, 12:369-381.

31. Arking DE, Chugh SS, Chakravarti A, Spooner PM: Genomics in sudden cardiac death. Circ Res 2004, 94:712-723.

32. Myerburg RJ, Castellanos A: Emerging paradigms of the epidemiology and demographics of sudden cardiac arrest. Heart Rhythm 2006, 3:235-239.

33. Noseworthy PA, Newton-Cheh C: Genetic determinants of sudden cardiac death. Circulation 2008, 118:1854-1863.

34. •• Bezzina CR, Pazoki R, Bardai A, et al.: Genome-wide association study identifies a susceptibility locus at $21 \mathrm{q} 21$ for ventricular fibrillation in acute myocardial infarction. Nat Genet 2010 (in press). This is the first case-control genome-wide association study carried out in patients with a first acute MI aimed at identifying common genetic variants associated with VF during acute ischemia. This study uncovered SNPs at the CXADR locus associated with $V F$.

35. Bowles NE, Richardson PJ, Olsen EG, Archard LC: Detection of Coxsackie-B-virus-specific RNA sequences in myocardial biopsy samples from patients with myocarditis and dilated cardiomyopathy. Lancet 1986, 1:1120-1123.

36. Pauschinger M, Bowles NE, Fuentes-Garcia FJ, et al. : Detection of adenoviral genome in the myocardium of adult patients with idiopathic left ventricular dysfunction. Circulation 1999, 99:1348-1354.

37. Andreoletti L, Venteo L, Douche-Aourik F, et al.: Active Coxsackie viral $\mathrm{B}$ infection is associated with disruption of dystrophin in endomyocardial tissue of patients who died 
suddenly of acute myocardial infarction. J Am Coll Cardiol 2007, 50:2207-2214.

38. • Lisewski U, Shi Y, Wrackmeyer U, et al.: The tight junction protein CAR regulates cardiac conduction and cell-cell communication. J Exp Med 2008, 205:2369-2379. Through studies in inducible Coxsackievirus and adenovirus receptor (CAR) knockout mice, these investigators reported a physiologic role for CAR in atrioventricular conduction. This study also showed that loss of CAR was accompanied by downregulation of connexin 45 and connexin 43 at the intercalated disk.

39. • Lim BK, Xiong D, Dorner A, et al.: Coxsackievirus and adenovirus receptor (CAR) mediates atrioventricular-node function and connexin 45 localization in the murine heart. J Clin Invest 2008, 118:27582770. This study reported a role for the Coxsackievirus and adenovirus receptor (CAR) in atrioventricular conduction through studies in cardiac-specific CAR knockout mice and global CAR knockout embryonic hearts. This study also demonstrated that localization of connexin 45 at the $A V$-node cell-cell junction and of $\beta$-catenin and $\mathrm{ZO}-1$ at the ventricular intercalated disc are dependent on CAR.

40. Havlik RJ, Garrison RJ, Fabsitz R, Feinleib M: Variability of heart rate, P-R, QRS and Q-T durations in twins. J Electrocardiol 1980, 13:45-48.

41. Busjahn A, Knoblauch H, Faulhaber HD, et al.: QT interval is linked to 2 long-QT syndrome loci in normal subjects. Circulation 1999, 99:3161-3164.

42. Schouten EG, Dekker JM, Meppelink P, et al.: QT interval prolongation predicts cardiovascular mortality in an apparently healthy population. Circulation 1991, 84:1516-1523.

43. Straus SM, Kors JA, De Bruin ML, et al. : Prolonged QTe interval and risk of sudden cardiac death in a population of older adults. $\mathrm{J}$ Am Coll Cardiol 2006, 47:362-367.

44. Vrtovec B, Delgado R, Zewail A, et al.: Prolonged QTe interval and high B-type natriuretic peptide levels together predict mortality in patients with advanced heart failure. Circulation 2003, 107:1764-1769.

45. Arking DE, Pfeufer A, Post W, et al.: A common genetic variant in the NOS1 regulator NOS1AP modulates cardiac repolarization. Nat Genet 2006, 38:644-651.

46. - Newton-Cheh C, Eijgelsheim M, Rice KM, et al.: Common variants at ten loci influence QT interval duration in the QTGEN Study. Nat Genet 2009, 41:399-406. This meta-analysis of three genome-wide association studies in individuals of European ancestry uncovered multiple novel common genetic variants associated with QT interval in general population.

47. - Pfeufer A, Sanna S, Arking DE, et al.: Common variants at ten loci modulate the QT interval duration in the QTSCD Study. Nat Genet 2009, 41:407-414. This report analyzed genome-wide data from five population-based cohorts in individuals of European ancestry and identified multiple novel loci associated with the QT interval.

48. - Crotti L, Monti MC, Insolia R, et al.: NOS1AP is a genetic modifier of the long-QT syndrome. Circulation 2009, 120:1657-
1663. This family-based association study carried out in LQTS families segregating the KCNQ1 A341V founder mutation (205 mutation carriers, 228 non-carriers), demonstrated for the first time that NOSIAP SNPS modify disease expression (QT interval and arrhythmia risk) in this disorder.

49. - Tomás M, Napolitano C, De GL, et al.: Polymorphisms in the NOS1AP gene modulate QT interval duration and risk of arrhythmias in the long QT syndrome. J Am Coll Cardiol 2010, 55:2745-2752. This cohort study carried out 901 patients from 520 families with LQTS caused by mutations in different genes provided evidence for a role of SNPS in the NOS1AP gene in modulation of QT interval duration and risk of arrhythmias in this disorder.

50. Kao WH, Arking DE, Post W, et al.: Genetic variations in nitric oxide synthase 1 adaptor protein are associated with sudden cardiac death in US white community-based populations. Circulation 2009, 119:940-951.

51. - Holm H, Gudbjartsson DF, Arnar DO, et al.: Several common variants modulate heart rate, PR interval and QRS duration. Nat Genet 2010, 42:117-122. This report entailed a genome-wide association study in individuals of European descent that uncovered multiple common genetic variants associated with heart rate and ECG indices of conduction (PR- and QRS-interval).

52. - Chambers JC, Zhao J, Terracciano CM, et al.: Genetic variation in SCN10A influences cardiac conduction. Nat Genet 2010, 42:149-152. This report entailed a genome-wide association study in Asians and Europeans that identified SNPS in SCN10A modulating cardiac conduction.

53. - Pfeufer A, van NC, Marciante KD, et al:: Genome-wide association study of PR interval. Nat Genet 2010, 42:153-159. This report entailed a meta-analysis of genome-wide association studies for PR interval from multiple population-based European studies that revealed multiple common genetic variants associated with this trait.

54. Schott JJ, Alshinawi C, Kyndt F, et al.: Cardiac conduction defects associate with mutations in SCN5A. Nat Genet 1999, 23:20-21.

55. Jay PY, Harris BS, Buerger A, et al.: Function follows form: cardiac conduction system defects in Nkx2-5 mutation. Anat Rec A Discov Mol Cell Evol Biol 2004, 280:966-972.

56. Moskowitz IP, Kim JB, Moore ML, et al.: A molecular pathway including Id2, Tbx5, and Nkx2-5 required for cardiac conduction system development. Cell 2007, 129:1365-1376.

57. Mesbah K, Harrelson Z, Theveniau-Ruissy M, et al.: Tbx3 is required for outflow tract development. Circ Res 2008, 103:743750.

58. Postma AV, van de Meerakker JB, Mathijssen IB, et al.: A gain-offunction TBX5 mutation is associated with atypical Holt-Oram syndrome and paroxysmal atrial fibrillation. Circ Res 2008, 102:1433-1442.

59. Schinzel A: Ulnar-mammary syndrome. J Med Genet 1987, 24:778-781. 